\title{
Overuse of Primary Thromboprophylaxis in Medical Inpatients at Low Risk of Venous Thromboembolism
}

J Gen Intern Med 36(9):2883-5 DOI: $10.1007 / \mathrm{s} 11606-020-05993-\mathrm{x}$

(C) Society of General Internal Medicine (This is a U.S. government work and not under copyright protection in the U.S.; foreign copyright protection may apply) 2021

\section{INTRODUCTION}

Venous thromboembolism (VTE) is a common cause of morbidity and mortality. ${ }^{1}$ Hospitalization and acute medical illness increase the risk of VTE, and hospital-associated VTE represents a preventable cause of morbidity and mortality. ${ }^{1}$ Accordingly, accreditation and regulatory agencies endorse inpatient pharmacologic VTE prophylaxis (PPX) as a quality measure. ${ }^{2}$ To raise rates of PPX prescribing, many health systems have adopted a default approach to electronic ordering, in which clinicians must "opt-out" of PPX prescription. ${ }^{2}$ However, this strategy may cause medical overuse and avoidable harms, prompting the American Society of Hematology (ASH) to recommend a risk-adapted approach to PPX. ${ }^{3}$ One validated risk model endorsed by ASH is the IMPROVE-VTE risk assessment model ${ }^{4,}$, , which can identify patients who are at low risk for VTE and may not warrant pharmacologic PPX. We therefore sought to compare the actual practice of PPX prescribing to the guideline-recommended strategy according the IMPROVE-VTE model in a large population of medical inpatients.

\section{METHODS}

In this observational study, we used electronic health record (EHR) data to identify adult, medical inpatients hospitalized at Yale-New Haven Hospital (a 1541-bed, urban, and academic medical center in New Haven, CT, that includes two hospital sites located approximately 1 mile apart) from Jan. 2014 to Dec. 2018. We excluded patients who were admitted to non-medical services, pregnant, admitted for VTE or bleeding, prescribed full-dose anticoagulation or direct oral anticoagulants (at any dose) on admission, or had a platelet count of $<50,000 / \mu \mathrm{L}$. The International Review Board at Yale University deemed this study exempt from full review.

Received May 24, 2020

Accepted June 12, 2020

Published online January 19, 2021
For each included patient, we determined VTE risk by calculating their IMPROVE-VTE score on admission using the previously validated model weights. ${ }^{4,5} \mathrm{We}$ chose to assess risk at the time of admission since this is typically when PPX ordering occurs. For each component other than age, we used ICD-10 codes that were billed either prior to or upon admission to determine the presence of these risk factors. In accordance with the ASH guidelines, we used an IMPROVE-VTE score of $<2$ to differentiate patients at low risk of hospitalassociated VTE from those at higher risk (IMPROVE score $\geq$ 2). We used inpatient medication order history data to determine receipt of PPX.

\section{RESULTS}

We identified 126,634 patients hospitalized during the 5year study period, of which 90,429 met inclusion criteria (see Fig. 1). The average age was 63 years (standard deviation 18 years); $52 \%$ were female and $32 \%$ were non-White (see Table 1). Of the included patients, 69\% were at low risk of VTE (of these, $39 \%$ had an IMPROVE-VTE score of 0 and $61 \%$ had a score of 1) and $31 \%$ were at higher risk (IMPROVE-VTE $\geq 2$ ). Within the low-risk group, $86 \%$ received PPX (of these, 67\% received unfractionated heparin and 19\% received low molecular weight heparin).

\section{CONCLUSIONS}

In this contemporary cohort of adult, medical inpatients, we found that the majority of patients $(69 \%)$ were at low risk of hospital-associated VTE $(\leq 0.5 \%$ risk within 3 months of $\operatorname{admission}^{3}$ ). Among these low-risk patients, $86 \%$ received pharmacologic PPX, representing a group in whom PPX may be unnecessary. Our results are consistent with another study $^{6}$ that found $78 \%$ of patients at low risk for VTE (using the Padua Prediction Score) received excessive PPX. Using a risk-adapted approach, rather than default PPX ordering, may therefore reduce medical overuse and avoidable harms.

Owing to its retrospective design, a key limitation of our study is the exclusive use of data available in the EHR to determine each patient's risk of VTE. If any conditions that comprise the IMPROVE-VTE score were present but not 


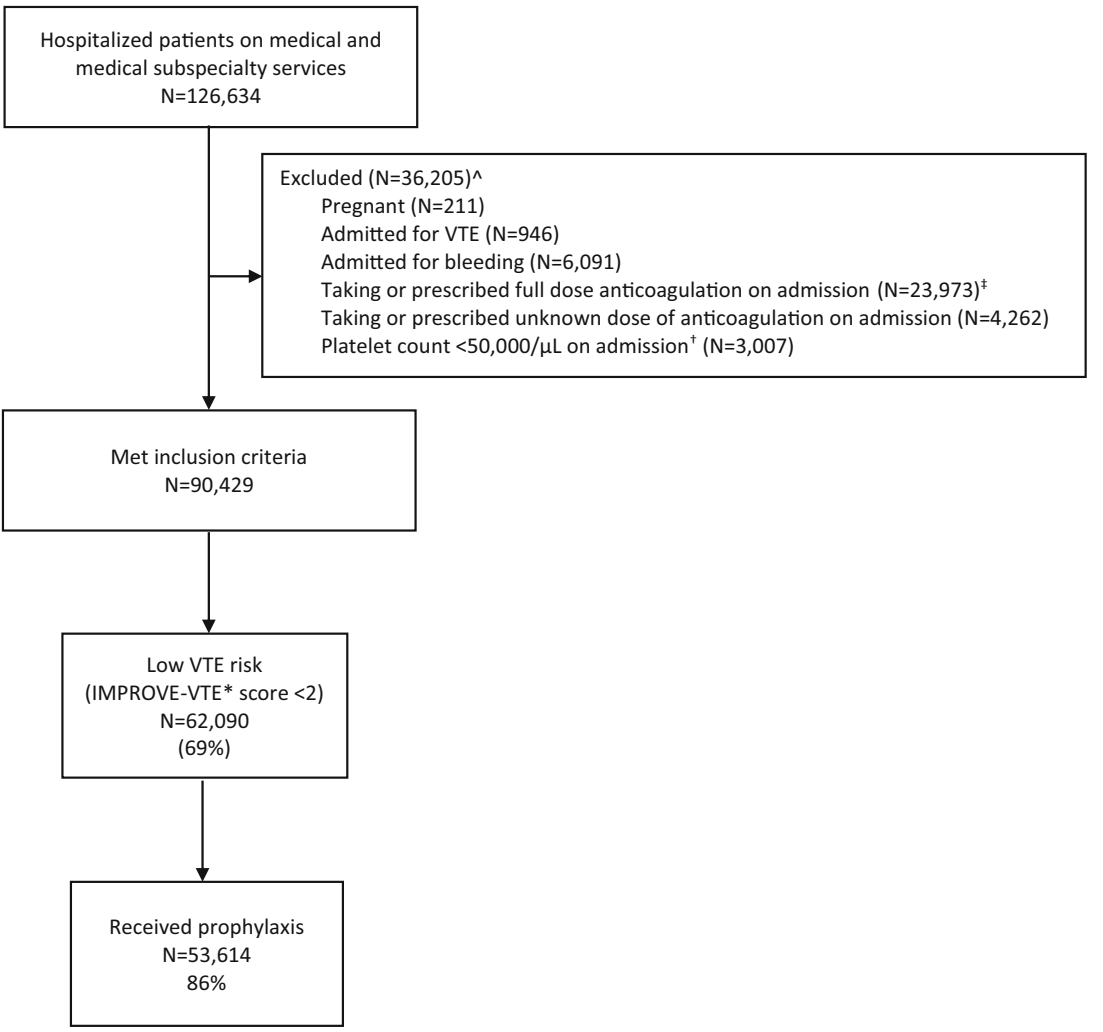

Figure 1 Flow chart of patient selection and prophylaxis frequency.

Legend: prophylaxis= pharmacologic prophylaxis; $\mathrm{VTE}=$ venous thromboembolism

^ Patients could meet more than one exclusion criterion.

\$ determined if the first anticoagulant ordered during the hospitalization was not unfractionated heparin, low-molecular weight heparin or fondaparinux at prophylactic dose

$\dagger$ used the first value available within the $1^{\text {st }} 24$ hours of admission. If no platelet value was provided within 24 hours of admission, we did not exclude the patient based on this criterion

* IMPROVE-VTE score: a risk assessment model that calculates the risk of hospital-associated VTE in hospitalized medical patients. For each patient, we calculated the IMPROVE-VTE score using the previously validated model weights: 3 points for a prior history of VTE; 2 points for known thrombophilia, lower limb paralysis, or active cancer; 1 point for immobilization, admission to the intensive care unit, or age $\geq 60$ years.

adequately billed or coded for, then this would have led us to underestimate the VTE risk. Another limitation of our study is that we did not ascertain bleeding as a complication of unnecessary PPX; there is not a validated and reliable method of doing so using EHR data. Nevertheless, overuse of injectable PPX can affect patient comfort and experience. We were also limited by studying only one hospital system, though we did include two hospitals.

Overall, there appears to be over-utilization of PPX among medical inpatients at low risk of hospital-associated VTE, wrhich warrants re-evaluation of PPX-prescribing practices.

Table 1 Characteristics of Study Participants $(N=90,429)$

\begin{tabular}{ll}
\hline \hline Variable & \\
Age, mean (SD) years & $63(18)$ years \\
Female, $N(\%)$ & $47,028(52 \%)$ \\
Non-White, $N(\%)$ & $28,804(32 \%)$ \\
Hispanic, $N(\%)$ & $9453(10 \%)$ \\
Admitted to & \\
General medicine & $55,532(61 \%)$ \\
Subspecialty floor & \\
Step-down unit or intensive care unit & $21,997(24 \%)$ \\
\hline
\end{tabular}

† Subspecialty floors include inpatient hematology, oncology, cardiology, and hepatology
Acknowledgments: The authors gratefully acknowledge Richard Hintz from the Yale-New Haven Hospital Joint Data Analytics Team for facilitating data acquisition.

Mia Djulbegovic, $M D^{1,2,3}$

Kevin Chen, $\mathrm{MD}^{1,2,3}$

Soundari Sureshanand, $M C A^{4,5}$

Sarwat Chaudhry, $\mathrm{MD}^{1,3}$

${ }^{1}$ National Clinician Scholars Program, Yale University School of Medicine,

New Haven, CT, USA

${ }^{2}$ Veterans Affairs Connecticut Healthcare System,

West Haven, CT, USA

${ }^{3}$ Department of Internal Medicine, Yale University School of Medicine,

New Haven, CT, USA

${ }^{4}$ Joint Data Analyst Team, Yale University,

New Haven, CT, USA

${ }^{5}$ Yale Center for Clinician Investigation,

New Haven, CT, USA

Corresponding Author: Mia Djulbegovic, MD; National Clinician Scholars Program, Yale University School of Medicine, New Haven, CT, USA (e-mail: mia.djulbegovic@yale.edu). 
Funding Financial support was provided to Dr. Mia Djulbegovic and Dr. Kevin Chen by CTSA Grant Number TL1 TR001864 from the National Center for Advancing Translational Science (NCATS), a component of the National Institutes of Health (NIH). These funds were provided to Drs. Djulbegovic and Chen by the National Clinician Scholars Program at Yale University School of Medicine. Dr. Djulbegovic and Dr. Chen are also sponsored by the Veterans Affairs Connecticut Healthcare System.

\section{Compliance with Ethical Standards:}

The International Review Board at Yale University deemed this study exempt from full review.

Conflict of Interest: Dr. Sarwat Chaudhry is a Paid Reviewer for the CVS State of CT Clinical Pharmacy Program.

Disclaimer: The content of this work is solely the responsibility the author team and do not necessarily represent the official view of NIH.

\section{REFERENCES}

1. Heit JA, Spencer FA, White RH. The epidemiology of venous thromboembolism. J Thromb Thrombolysis. 2016;41:3-14.
2. Maynard G. Preventing Hospital-Associated Venous Thromboembolism: A Guide for Effective Quality Improvement. AHRQ Publication No. 16-001EF. 2016.

3. Schünemann HJ, Cushman M, Burnett AE, et al. American Society of Hematology 2018 guidelines for management of venous thromboembolism: prophylaxis for hospitalized and nonhospitalized medical patients. Blood Adv. 2018;2(22):3198-3225.

4. Mahan CE, Liu Y, Turpie AG, et al. External validation of a risk assessment model for venous thromboembolism in the hospitalised acutely-ill medical patient (VTE-VALOURR). Thromb Haemost. 2014;112(04):692-699.

5. Rosenberg D, Eichorn A, Alarcon M, McCullagh L, McGinn T, Spyropoulos AC. External validation of the risk assessment model of the International Medical Prevention Registry on Venous Thromboembolism (IMPROVE) for medical patients in a tertiary health system. J Am Heart Assoc. 2014;3(6):e001152.

6. Grant PJ, Conlon A, Chopra V, Flanders SA. Use of venous thromboembolism prophylaxis in hospitalized patients. JAMA Inter Med. 2018;178(8):1122-1124.

Publisher's Note: Springer Nature remains neutral with regard to jurisdictional claims in published maps and institutional affiliations. 